\title{
Estudio de factibilidad del aprovechamiento económico de una especie invasora Hypostomus plecostomus en el humedal de Caño Negro, Costa Rica
}

\section{Feasibility study of the economic exploitation of the invasive species Hypostomus plecostomus in the Caño Negro Wetlands, Costa Rica}

\author{
Raúl Fonseca-Hernández ${ }^{1 *}$ y Paulina Vargas-Alpízar ${ }^{2}$
}

\begin{abstract}
RESUMEN
El Refugio Nacional de Vida Silvestre Mixto Caño Negro se ubica entre los cantones de Guatuso y Los Chiles en Alajuela, Costa Rica. El humedal que se presenta en este Refugio tiene una presencia abundante de la especie invasora denominada Hypostomus plecostomus o pez pleco, la cual afecta al ecosistema nativo y genera consecuencias socioeconómicas para los habitantes de la zona. El objetivo de este estudio fue evaluar la factibilidad técnica y financiera de una alternativa para el aprovechamiento económico de la especie invasora. Se aplicó la revisión secundaria de datos, encuestas, entrevistas a expertos y trabajo de campo. La alternativa productiva analizada es la elaboración de harina de pescado para consumo animal (vacuno de engorde). El proyecto posee una sólida justificación, con resultados positivos en el estudio técnico y financiero, donde se obtiene una tasa interna de retorno del 2\%, y valor actual neto de los ingresos $₫ 35729676$. Adicionalmente, se generará empleo en la zona. Sin embargo, el componente legal frena, en el corto plazo, la ejecución del proyecto, dado que, en la actualidad, en cuerpos de agua continentales, solo se permite la pesca por medio de cuerda y el pez pleco no puede ser capturado mediante esta, por lo que se requeriría una modificación a la Ley actual. El trabajo generó una metodología base hacia el análisis futuro, para la evaluación de proyectos productivos que busquen el aprovechamiento económico, cuyas poblaciones requieran ser controladas por sus efectos negativos en los ecosistemas locales.
\end{abstract}

Palabras clave: Hypostomus plecostomus, aprovechamiento económico, especie invasora, plecos, Caño Negro

\section{ABSTRACT}

The Caño Negro Mixed National Wildlife Refuge is located between Guatuso and Los Chiles counties in Alajuela, Costa Rica. The Caño Negro Wetlands has an abundant presence of the

1 Economista, académico e investigador de la Escuela de Relaciones Internacionales de la Universidad Nacional y de la Sección de Economía de Sede de Occidente, Universidad de Costa Rica, raul.fonseca.hernandez@una.cr*

2 Bióloga, Proyecto Humedales, Sistema Nacional de Áreas de Conservación, paulina.vargas@sinac.go.cr 
invasive species Hypostomus plecostomus or suckermouth catfish (locally known as pleco fish), which affects the native ecosystem and generates socioeconomic consequences for the inhabitants of the area. This paper is aimed at evaluating the technical and financial feasibility of an alternative for the economic exploitation of the invasive species. Secondary data analysis, surveys, fieldwork, and expert interviews were applied. The most feasible alternative is the production of fish flour for cattle fattening. The project has a strong technical and financial justification that supports its feasibility, with a $2 \%$ internal rate of return and a net present value of income equivalent to $₫ 35,729,676$. In addition, this option will generate employment in the area. However, the legal component is stopping the execution of the project in the short term since, at present, the only fishing allowed in continental waters is by means of rods or ropes, which would require a modification to the current law as pleco fish cannot be caught this way. This research study generated a baseline methodology for future analysis for the evaluation of profitable projects aiming to generate economic exploitation of populations that require to be controlled due to their negative effects on local ecosystems.

Keywords: Hypostomus Plecostomus, economic exploitation, invasive species, plecos, Caño Negro

\section{INTRODUCCIÓN}

El aprovechamiento económico de especies invasoras presentes en áreas bajo alguna categoría de manejo puede ser una fuente de empleo para las comunidades aledañas y una opción viable para contener y mermar los efectos negativos sobre el ecosistema. Esta discusión puede darse al comprobar la existencia de una especie en una avanzada etapa de invasión, lo cual implica que la especie ya ha colonizado el hábitat en estudio y se reproduce sin problemas, y contraponer los daños que esta puede generar al ecosistema local frente a un proyecto productivo que esté acorde con el entorno de la comunidad y aproveche como insumo la especie invasora.

El sitio de estudio es el Refugio Nacional de Vida Silvestre Mixto Caño Negro (RNVSMCN), el cual posee registros recientes de la existencia de una población importante de Hypostomus plecostomus o peces pleco, que son pertenecientes a la familia Loricariidae, los cuales son catalogados tanto en Costa Rica, como en otros países como peces exóticos e invasores que provocan daños a especies nativas, ecosistemas y economías locales (Mendoza et al. 2007; Nico et al. 2009a; Ayala-Pérez et al. 2014). Así mismo, las comunidades aledañas tienen condiciones de vulnerabilidad social agravadas por la falta de opciones de empleo en la zona, aspecto que podría mejorar con el aporte económico del pez.

La investigación se dio en el marco de un trabajo conjunto realizado por el Proyecto Conservación, uso sostenible de la biodiversidad y mantenimiento de los servicios de los ecosistemas de humedales protegidos 
de importancia internacional (conocido como Proyecto Humedales), con el auspicio del Fondo para el Medio Ambiente Mundial (GEF), administrado por el Programa de las Naciones Unidas para el Desarrollo (PNUD) e implementado por el Sistema Nacional de Áreas de Conservación (SINAC) (Proyecto Humedales, 2017). Lo cual permitió el intercambio disciplinario de la biología, en conjunto con la economía, para la realización del análisis en cuestión.

El objetivo de este trabajo fue evaluar la factibilidad técnica y financiera de un emprendimiento productivo, que utilice como insumo la especie invasora $H$. plecostomus o pez pleco, presente en el humedal de Caño Negro, para la generación de alternativas productivas que permitirían a las comunidades aledañas mejorar sus condiciones de vida, así como la minimización del impacto que dicha especie genera en el humedal. Se busca atender las siguientes interrogantes: ¿Cuáles elementos debe considerar un proyecto productivo para ser técnica y financieramente viable?, así como ¿Cuál será el aprovechamiento económico más viable que utilice como insumo la especie invasora pez pleco en el humedal de Caño Negro?

\section{MATERIALES Y MÉTODOS}

La investigación tiene un método cuantitativo, bajo un enfoque de factibilidad técnica y de demanda. Se realizó en dos etapas. En la primera se constata mediante fuentes bibliográficas complementarias y en la segunda etapa se realizó trabajo de campo para elegir el aprovechamiento más adecuado que se le podría dar al pez pleco. En cuanto a las referencias bibliográficas, se contó con una importante limitación, dado que sobre el tema en estudio existe un acervo documental restringido en cuanto abordar el tema desde dos perspectivas: la biológica y la económica, por lo que se recurre a utilizar referencias bibliográficas con una antigüedad mayor a los cinco años.

Para la segunda etapa se realizaron ocho giras a la zona de estudio y alrededores, entre enero y julio de 2017. Las giras de trabajo, con un grupo de aproximadamente 20 vecinos representantes de las cuatro asociaciones de desarrollo integral existentes en el distrito de Caño Negro, estas son: Caño Negro, Nueva Esperanza, Buenos Aires y Veracruz, quienes potencialmente se encargarían de la ejecución y administración del emprendimiento.

La elección de la idea productiva que podría dar un aprovechamiento económico a la especie invasora fue seleccionada mediante una búsqueda de datos secundarios y un levantamiento primario de información con el grupo de vecinos de la zona. El levantamiento primario de información se realizó mediante el método constructivista y la herramienta del metaplan. De tal forma que se partió de las 
experiencias y conocimientos previos de las personas participantes (Tobón, 2008) en el manejo de otros peces y el conocimiento de la cultura local, para determinar los posibles usos de la especie invasora en particular. Los mismos fueron confrontados con los resultados obtenidos en la literatura y en las entrevistas al médico veterinario Carlos Alpízar Solís de la Escuela de Medicina Veterinaria de la Universidad Nacional (Alpízar, comunicación personal, marzo 20, 2017), así como Nelson Peña Navarro (Peña, comunicación personal, enero 14, 2017) coordinador de la Unidad de Investigación de la Universidad Técnica Nacional y desarrollo comunitario de la Universidad Técnica Nacional.

Por último, la tercera etapa consistió en el análisis de la viabilidad técnica y económica del aprovechamiento de la especie invasora. Esto se realizó mediante el método de plan de negocios y evaluación financiera de proyectos. El desarrollo de los principios de un plan de negocios básico de Pedraza (2011) sigue las siguientes partes:

- Justificación: la cual consiste en la identificación de las razones que motivan el desarrollo de la idea de negocios.

- $\quad$ Estudio de mercado: búsqueda de información secundaria, entrevistas a profundidad según las actividades descritas en la segunda etapa del proyecto.

Estudio técnico: permite conocer la viabilidad técnica del proyecto. Analiza la escala de planta (tamaño requerido para la producción pretendida), los materiales, insumos y maquinaria requeridos para la producción como tal, el tipo y diseño de infraestructura, la cantidad de recurso humano, la direccionalidad del flujo de proceso así como los tiempos de producción, entre cualquier otro elemento necesario para poner en marcha el proyecto. Este apartado se elaboró con base en las entrevistas a profundidad.

El estudio financiero: se genera el presupuesto del proyecto al sumar los costos asociados y se planifica el flujo de caja del mismo (Valverde \& Alfaro, 2014). Se aplica un tipo de análisis financiero basado en el cálculo e interpretación de las siguientes razones financieras para analizar su viabilidad: Tasa Interna de Retorno y el Valor Actual Neto de la Inversión (Sapag, 2007; Zequeira et al. 2017) 


\section{RESULTADOS Y DISCUSIÓN}

A continuación, se desarrollan los siguientes componentes: justificación, estudio de mercado, estudio técnico y estudio financiero, como los resultados obtenidos por la investigación. Por lo tanto, en cada componente dichos resultados darán a conocer la viabilidad técnica y económica de la propuesta productiva para aprovechar económicamente la especie invasora en el humedal de Caño Negro.

\section{Justificación de la propuesta}

El proyecto se justifica en tres aspectos primordiales: el primero parte de un razonamiento biológico, al considerar la presencia abundante de una especie invasora en el humedal de Caño Negro; el segundo es socioeconómico, vinculado a las condiciones de vulnerabilidad social que tienen los habitantes de la zona. El tercer aspecto es la elección de la idea de negocio, mediante un proceso investigativo y participativo con la comunidad, lo cual lo hace adecuado al contexto particular y viabiliza su posterior aplicación. A continuación, se desarrolla cada uno de estos elementos:

La especie exótica presente en el humedal es conocida popularmente como pez pleco o diablo. Proviene del orden Siluriformes, de la familia denominada Loriicaridae, la cual se encuentra usualmente en la costa pacífica en el Sur de América y Centroamérica (Guerrero, 2016). Según Guerrero (2016), existen más de 700 especies para esta familia y su taxonomía es compleja. Se caracterizan por un cuerpo deprimido cubierto por placas óseas, un par único de barbillas maxilares y, sobre todo, por una modificación importante de la estructura de la boca en un disco de ventosa, lo que les permite que se adhieran al sustrato, incluso en aguas de flujo particularmente rápido. La boca y los dientes muestran fuertes adaptaciones a la alimentación, condicionados para raspar algas, pequeños invertebrados, detritus e incluso madera sobre sustratos sumergidos (Covain y Fisch-Muller, 2007). Existe controversia sobre el nombre científico a utilizar para la especie presente en Caño Negro, Angulo et al. (2013) y Angulo (2013) le identifican como Hemiancistrus aspidolepis, sinónimo de Hypostomus panamensis.

Como lo estipula el Artículo 31 del Reglamento a la Ley de Conservación de la Vida Silvestre, para que una especie sea catalogada como invasora debe existir una declaratoria por parte del SINAC, fundamentada en cierta información técnica. Actualmente el SINAC no ha realizado esta declaratoria para el pez pleco, pero desde nuestra perspectiva cumpliría a cabalidad con lo solicitado en dicha norma. Esta es una especie exótica para la región y se encuentra en un estado avanzado de invasión de acuerdo con las etapas 
citadas por Blackburn et al. (2011), los peces encontrados corresponde a tal es el caso de la población de pez pleco de Caño Negro y alrededores, pues se ha reportado en todos los ríos de la subvertiente norte (observación personal) y Caribe (Herrera \& Molina, 2011), así como en el lago de Nicaragua (Corea et al. 2014).

En el marco del proyecto $\mathrm{Hu}-$ medales, Chacón (2016) realizó un muestreo, que si bien es preliminar, proporciona evidencia importante sobre la abundancia del pez pleco en varios segmentos del humedalde este espacio, y corrobora su avanzado estado de invasión (Cuadro 1). En los dos sitios muestreados sobre el río Frío, el porcentaje promedio de peces diablo respecto al total colectado fue de $25 \%$, lo que significa que un cuarto de todos peces exóticos, además es común encontrarlos y este dato representa una primera voz de alerta sobre la invasión, ya que es un porcentaje mayor que el de muchas familias nativas. Las experiencias con Loricariidos en otros países han demostrado su fuerte potencial invasor (Wakida-Kusunoki y Amador del Angel, 2008; Corea et al. 2014; Mendoza et al. 2007). El trabajo del Proyecto Humedales de Chacón (2016) sirve de base para la realización del presente estudio.

Las características fisiológicas, morfológicas y de comportamiento explican su potencial invasivo, según Guerrero (2016) la familia posee una alta tasa de reproducción larval y hábitos de anidación nocturna. Así mismo,

Cuadro 1. Características biométricas y estimaciones de densidad poblacional del $H$. plecostomus o pez pleco en el río Frío, RNVSM Caño Negro, 2016

Table 1. Biometric characteristics and population density estimation of $H$. plecostomus or pleco fish in the Frio River, Caño Negro MNWR, 2016

\begin{tabular}{lcc}
\hline \multicolumn{1}{c}{ Lugar } & $\begin{array}{c}\text { Río Frío } \\
\text { Segmento }\end{array}$ & $\begin{array}{c}\text { Río Frío } \\
\text { Segmento 2 }\end{array}$ \\
\hline Porcentaje de plecos capturados (\%)* & 32.00 & 19.00 \\
Razón no peces / Lance & 2.86 & 0.78 \\
Densidad peces por área (peces $\left./ \mathrm{m}^{2}\right)$ & 0.62 & 0.17 \\
Peso promedio $(\mathrm{g})$ de pleco & 146.4 & 269.5 \\
Longitud total promedio $(\mathrm{cm})$ & 24.3 & 30.0 \\
Densidad por Km ${ }^{2}$ & 12400 & 3400 \\
kg de peces $/ \mathrm{km} \mathrm{lineal}$ & 1815 & 916 \\
\hline
\end{tabular}

Fuente: Elaboración propia con base en los resultados obtenidos por Chacón (2016)

*En relación con la abundancia de peces de las familias locales. 
tienen pocos depredadores, dado sus características físicas.

Los efectos que esta especie puede causar en un ecosistema son diversos, e incluso puede producir fuertes impactos en la economía local. Seguidamente se enumeran las principales consecuencias negativas que puede generar esta especie:

1) Amenazar la supervivencia de las especies autóctonas y de importancia comercial, dada la importante competencia directa por espacio y alimento (Mendoza et al. 2007; Nico et al. 2009a; Ayala-Pérez et al. 2014; Chacón, 2016).

2) Se especula que, por sus hábitos alimenticios, podría estar depredando estadios tempranos (al consumir huevos y larvas) de varias especies no solo de peces nativos, sino de macroinvertebrados, lo que supone un severo problema de reproducción para estas especies (Mendoza et al. 2007).

3) Por sus hábitos y la construcción de madrigueras en forma de galerías profundas en las paredes de los cuerpos de agua, se le hace responsable del deterioro del borde del río, lo que provoca erosión. Se estima que los plecos pueden ocasionar hasta cuatro metros de erosión al año (Nico et al. 2009b). Dichas galerías han sido documentadas en el humedal de Caño Negro por Chacón (2016).

4) Se han reportado parásitos en diferentes especies de plecos (Sanz, 2010; Rodríguez-Santiago et al. 2016), pueden transferir e introducir patógenos, parásitos y enfermedades ajenas a las especies nativas, poniendo en riesgo la salud de las poblaciones locales (Martinez, 2007).

5) Desde el punto de vista socioeconómico, la presencia del pleco ha generado la disminución de las pesquerías tradicionales y de alto valor comercial en otros países (Mendoza et al. 2007).

En el estudio realizado por Chacón (2016) para el Proyecto humedales, entre los meses de marzo y abril de 2016, se puede determinar que existe una invasión avanzada de peces pleco en el río Frío, que alimenta el humedal de Caño Negro. El Cuadro 1 muestra los principales resultados del estudio realizado. En los segmentos del río Frío, se evidencia una considerable proporción de plecos respecto a otras familias de peces nativas del lugar, incluso este porcentaje de captura es superior a varias de estas familias locales. En el trabajo del Proyecto Humedales realizado por Chacón (2016) se encontraron hembras grávidas, además de gran cantidad de galerías para anidación de estos peces, por lo que se confirma la reproducción en la zona. 
De conformidad con lo anterior, se identifica un potencial de explotación responsable de la especie exótica pez pleco, que sería ventajoso para otras especies en el humedal de Caño Negro, como insumo para bienes transables en el mercado.

En un segundo orden de argumentos, se plantea la vulnerabilidad socioeconómica de la zona. En términos de pobreza, se registra en el nivel nacional que de cada 100 hogares, $77 \%$ se califica como no pobre mientras que el $22.4 \%$ como pobre, siendo de esta cifra un $15.7 \%$ como pobreza no extrema y el restante $6.7 \%$ pobreza extrema. Por su parte, en la Región Huetar Norte del país, de 100 hogares, $73.2 \%$ no es pobre, el restante $26.8 \%$ lo es, calificando un $17.3 \%$ en pobreza no extrema y un $9.6 \%$ en pobreza extrema (INEC, 2015), es decir, cifras mayores al promedio del país.

En el ámbito educativo, de acuerdo con el MIDEPLAN (2017), el porcentaje de analfabetismo es en promedio de $8.9 \%$ de la población en el caso del distrito de Los Chiles, y un 6\% en el caso del distrito de Caño Negro, siendo mayor en el caso de las mujeres con respecto a los hombres. En relación con el Índice de Desarrollo Humano 2014, el cantón de Los Chiles se encuentra en la última posición (N. ${ }^{\circ} 81$ ), marcando el 0.617 (PNUD, 2015).

Los bajos niveles de desarrollo económico y social que experimenta la región podrían explicarse por la falta de fuentes de empleo. La tasa de ocupación para el 2011 en Los Chiles y Caño Negro es del $45.7 \%$ y $42.7 \%$, respectivamente; inferior al promedio nacional $(51.7 \%)$. Por su parte, Caño Negro presenta una tasa de desempleo abierto relativamente baja, la cual es del $0.4 \%$ mientras que en Los Chiles es del $3.8 \%$, superior a la media nacional (3.4\%) (INEC, 2017).

Existen diversos usos comerciales que se le pueden dar al pez pleco y que han sido probados en diferentes países. Por ejemplo, en México se utilizan partes del pescado para realizar tratamientos odontológicos (Milenio, 2017), para la producción de galletas, diversos productos alimenticios (Informador, 2017), tales como filetes, concentrados proteínicos, complemento alimenticio para el ganado. Además, se da el aprovechamiento de la piel del pez para el desarrollo de artesanías como monederos, collares, aretes, entre otros (Medina, 2017). En Guatemala y Nicaragua se comercializa el pez en diferentes acuarios (El Nuevo Diario, 2010). En Perú se preparan ceviches y platillos con pez pleco (Castellón, 2017).

La producción de harina de pescado es otra alternativa de aprovechamiento del pez pleco según señalan diversos autores tales como Arroyo (2008), Escalera et al. (2012), Maldonado et al. (2015), (PRODAPESI, s. f.) y Valdez (2011), la harina de pescado es de alto valor nutricional como suple- 
mento alimenticio convencional, para ser utilizado en el crecimiento de aves, cerdos, rumiantes, vacas lecheras, ganado de engorde, ovino y camarón.

En el trabajo de campo se consultó al grupo de vecinos sobre posibles aprovechamientos del pez pleco, las siguientes fueron las propuestas mencionadas: alimento humano (ceviche, filete, chicharrón, empanizador de pescado, filetes empacados, chorizo, embutidos, platillos); alimento animales (concentrado para gatos, concentrado de pescado, carnada para pescar, harina para concentrado, harina, concentrado para animales de granja); producción industrial (abono/fertilizantes para plantaciones agrícolas o de uso doméstico); medicinal (producción de bacalao); manufactura (artesanías como collares, aretes, monederos, fajas, etc.); otros (campeonato de pesca, campeonato culinario, limpiador de peceras).

Dichas posibilidades se analizaron preliminarmente por sus requerimientos y características técnicas, capacidad de réplica, nivel de tecnificación, posibilidad de desarrollar en etapas, características de la población meta que desarrollaría el emprendimiento. Bajo estos criterios se determinó que la alternativa más viable sería elaborar harina de pescado para consumo animal. Dicho aprovechamiento económico posee las siguientes ventajas: experiencias de éxito en otros países que han implementado harina de pescado en la alimentación ganadera; agilización en el proceso logístico al tener el mercado regional; el proceso productivo puede hacerse de forma artesanal y poco tecnificado; la maquinaria utilizada tiene costos bajos; la población tiene experiencia en pesca; la inversión inicial es relativamente baja y de menor cuantía, en relación con otras ideas de negocio que se analizaron.

Estudio de mercado: Los resultados del estudio de mercado se obtuvieron de la aplicación de una encuesta a 17 ganaderos de la zona aledaña al humedal de Caño Negro, que podrían comprar la harina de pescado y 8 empresas productoras de alimentos para ganado. El estudio de mercado indagó sobre el tipo de alimento que utilizan en el proceso de engorde, sobresale el concentrado y la harina de pescado. Sin embargo, también se mencionan otros elementos como los pastos, la miel, melaza y yuca entre otros. Este tipo de insumos son adquiridos a proveedores como las Empresas Monteci1los, Dos Pinos y Agrologos, y a otros proveedores independientes.

El total de los encuestados manifestó que estarían dispuestos a adquirir la harina de pescado para su proceso de producción. Diversas razones fueron señaladas como justificantes para obtener este tipo de producto, entre ellas se mencionan el precio y los aportes nutricionales para el ganado, inclusive se menciona el hecho de ser, potencialmente, un sustituto de otros productos que ya se emplean en la ganadería. 
Con base en la encuesta aplicada se determina que se requieren alrededor de 30 sacos semanales de harina del pez pleco para abastecer la potencial demanda local.

Al entrevistar a las empresas que venden productos similares, se determina que el costo de los sacos de 46 kilogramos de harina de pescado es de 20520 colones en Aqua Corporación (Fernández, comunicación personal, julio 5, 2017) y 25992 colones en Prosalud (Salazar, comunicación personal, julio 5, 2017). Ambos establecimientos tienen capacidad industrial y están ubicados en Cañas y Puntarenas, respectivamente. Por tanto, no representan una competencia real para la empresa en cuestión. Se recomienda que el proyecto otorgue un precio de 20000 colones por saco. Este precio es considerado como razonable por los ganaderos y está por debajo del precio del mercado. Como se visualizará en el estudio financiero, este precio permite cubrir costos y obtener una ganancia para la actividad productiva.

Estudio técnico: El estudio técnico analiza los aspectos necesarios para hacer viable la idea de negocios sin considerar aún aspectos financieros, en este caso en particular, se consideran los siguientes cuatro elementos: en primera instancia se estudia el valor nutricional del pez pleco; en segundo término, se describe el tamaño de planta y los requerimientos específicos para la instalación de la fábrica. El tercero comenta las consideraciones legales vinculadas con la pesca de una especie invasora en un área protegida. El cuarto elemento muestra la proyección de crecimiento y prevalencia de la población del pez pleco en el humedal, aspecto relevante para garantizar el flujo constante de insumos para la fabricación de la harina.

En cuanto al valor nutricional, se muestra que el músculo de pleco de la zona norte tiene un alto valor nutricional y energético (calorías por porción), se le ubica, además, como una carne magra por su bajo contenido de grasa, lo que podrá proporcionar una cantidad adecuada de proteína al animal. Adicionalmente, tiene un aporte moderado de hierro y calcio.

En la entrevista realizada a Alpízar (2017) de la Escuela de Medicina Veterinaria de la Universidad Nacional, se pudo constatar que es un pescado que tendría un alto valor nutricional en la producción de harina para consumo animal. Se recomienda su utilización tanto para ganado de engorde como para el lechero. Sin embargo, se advierte que el lechero podría no ser un mercado en sí mismo, pues son las empresas que compran la leche las que suplen los concentrados y las fórmulas de alimentación a los ganaderos para lograr una uniformidad del producto. Por lo que se recomienda como complemento alimenticio del ganado de engorde.

Por otra parte, el proceso de producción se basaría en 5 etapas, 
las mismas fueron diseñadas por el Ingeniero Rodolfo Rivera Hernández, Gerente de Producción de una empresa trasnacional (Rivera, comunicación personal, mayo 14, 2017). En la primera etapa se recibirá el pescado, se pesará y registrará el ingreso para efectos contables y de producción, se entregaría comprobante a cada pescador para efectuar el pago posterior. La segunda etapa se inicia al pasar el pescado al área de corte, donde se reduce el tamaño de los animales para facilitar su posterior manipulación en el proceso. Cocinar el pescado a altas temperaturas y secarlo constituye la tercera etapa. Al finalizar este proceso, se tendrá una especie de masa seca. El siguiente paso es realizar manualmente la pulverización de la masa, de esta forma se obtendrá la harina de pescado para consumo animal. En la última y quinta etapa se empaca el producto, se etiqueta el empaque y se completan los pedidos a distribuir. La Figura 1, muestra el resumen del proceso productivo.

Para operar el proceso productivo descrito, se requiere un terreno, infraestructura, maquinaria y equipo, así como de un vehículo. El Cuadro 2, describe con mayor detalle los requerimientos necesarios para iniciar la producción artesanal de harina de pescado.

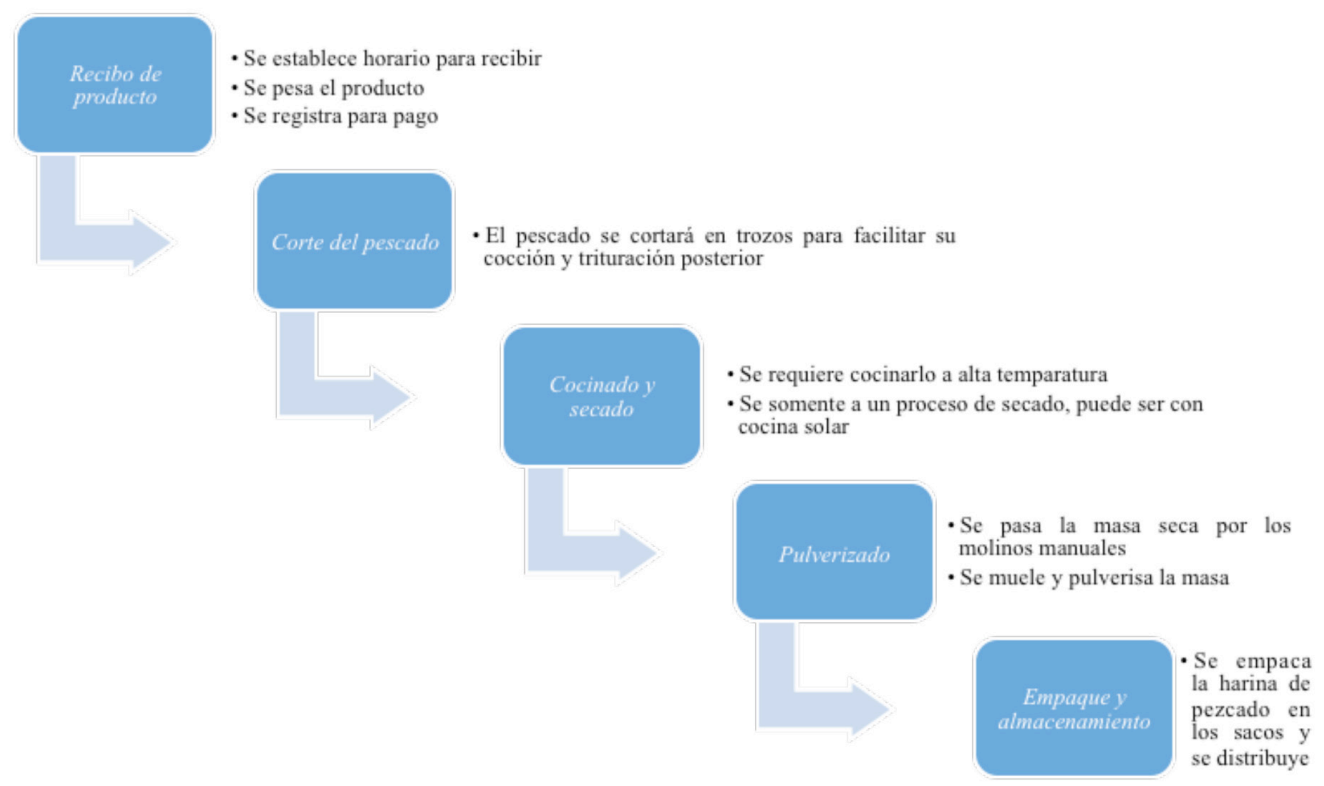

Fig. 1. Descripción del proceso de producción de harina de pescado Fuente: Elaboración propia con base a Rivera (2017)

Fig. 1. Description of fish flour production. Source: Adapted from Rivera (2017) 
Cuadro 2. Requerimientos para la construcción y equipamiento de la planta de producción de harina de pescado para consumo animal con base al $H$. plecostomus o pez pleco

Table 2. Requirements for the construction and equipment for the cattle fattening H. plecostomus or pleco fish flour production plant

\begin{tabular}{|c|c|}
\hline Requerimiento & Detalle \\
\hline Terreno & $\begin{array}{l}\text { Promedio entre } 1500 \text { y } 2000 \mathrm{~m}^{2} \text {. Se recomienda que esté si- } \\
\text { tuado con acceso o cercano al humedal. }\end{array}$ \\
\hline Edificación & $\begin{array}{l}\text { Consta de } 300 \text { metros cuadrados de construcción, tendrá un } \\
\text { galerón al frente para recibir el producto, así como áreas para } \\
\text { corte, cocinado, pulverizado y empaque. }\end{array}$ \\
\hline Equipo y maquinaria & $\begin{array}{l}\text { Incluye lo necesario para iniciar operaciones en una primera } \\
\text { etapa. }\end{array}$ \\
\hline Vehículo & Tipo pick-up, $4 \times 4$, con cajón \\
\hline
\end{tabular}

Para la operación del proyecto se requerirá un total de 25 trabajadores distribuidos de la siguiente forma: 20 pescadores certificados y 5 trabajadores en la planta (una persona que reciba el producto, una para el corte del pescado, dos para el proceso de secado y triturado, una para el proceso de empaque y distribución). Las personas trabajadoras se pagarán según la tasa establecida por el Ministerio de Trabajo, mientras que a los pescadores se les pagará 12 colones por pez promedio (146 g). Cada pescador tendrá un permiso que le autoriza para extraer alrededor de 1235 peces plecos por semana del humedal, siendo estos, los únicos acreditados para la venta del pescado a la Productora de Harina. La restricción se recomienda para una mejor organización y coor- dinación con las autoridades del SINAC responsables de fiscalizar los potenciales procesos de pesca, así como para garantizar el flujo de insumos necesarios y suficientes para cubrir la demanda inicial de 30 sacos de producto por semana.

El tercer elemento técnico a considerar es el aspecto legal. Es de vital importancia contar con un marco jurídico sólido que respalde todas las acciones a realizar. En este sentido, la Ley de Conservación de Vida Silvestre 7317 (LCVS) en su Artículo 22 establece que las especies que sean nativas o bien exóticas, y que estén causando afectaciones al ecosistema o a la sociedad en general, podrán ser sujetos de control o reubicación, según se disponga en el reglamento de la ley; por lo tanto, queda claro que se permite 
emprender acciones con las especies exóticas e invasoras.

Sin embargo, considerando que cualquiera de las formas de manejo dispuestas en el citado Artículo 22, supone realizar una acción de pesca, se deben considerar los artículos 67 y 68 de la misma Ley, que representa una limitante al proyecto de control y aprovechamiento del pez pleco. Este artículo menciona que, los métodos de pesca permitidos en cuerpos de agua continentales son exclusivamente: cuerda o caña, con anzuelo o engañador, y considerando que la modificación bucal que posee este pez no permite que se le capture por medio de cuerda o caña, se dificulta rotundamente su extracción. Además, la pesca con cuerda o caña, no es un método eficiente para captura masiva, que logre suplir la demanda local para la producción de harina y provocar así, un efecto positivo en el control de la población exótica.

Por lo tanto, es necesario realizar una modificación a la LCVS, la cual contemple una excepción a los métodos de pesca cuando se trate del control de especies invasoras. Aunado a lo anterior, el RNVSM Caño Negro posee una veda de pesca que deberá ser modificada para excluir la captura y control de los peces exóticos.

El cuarto y último elemento técnico a considerar es la proyección futura de la población del pez pleco, de tal forma que provea insumos suficientes para la producción de la harina de pescado. En este sentido, el proyecto requerirá de 24643 peces, considerando un peso promedio de 146 gramos por unidad, esto para la elaboración de 30 sacos de harina semanales. Para este cometido, se requeriría una población anual igual o mayor a 1182857 peces anuales. Según las proyecciones realizadas, basado en el estudio de Chacón (2016), la población estimada en los 25 kilómetros lineales que mide el río Frío dentro del área protegida se estimó en 31000 peces para el 2016, teniendo en cuenta una tasa de reproducción exitosa del $80 \%$, dado el cuido parental de la especie y la carencia de depredadores, y suponiendo que la mitad de esta población podrá aportar alrededor de 534 huevos anuales (observación personal) se estima que en dos años la población de peces pleco podría ser mayor a los 4000000 de individuos en la zona considerada del río Frío, en el humedal de Caño Negro. Bajo estas proyecciones y considerando la cantidad de peces potencialmente extraída anualmente con la población restante, se observa que existe capacidad para soportar la extracción anual de peces que requiere el proyecto productivo.

Estudio financiero: Para iniciar la producción de la harina del pez pleco para consumo animal se requieren los siguientes elementos: compra de terreno, construcción de instalaciones, contratación de la mano de obra para dicha construcción, costos de 
los permisos de construcción y elaboración de planos, compra de equipo, compra de vehículo, permisos para la producción, así como para la elaboración de trampas u otro método de pesca. El Cuadro 3 muestra el resumen de los costos aproximados.

Cuadro 3. Inversión inicial del proyecto calculada para junio 2017 -monto en colones

Table 3. Initial project investment estimated for June 2017, amount in Costa Rican Colones

\begin{tabular}{lr}
\hline \multicolumn{1}{c}{ Rubro } & \multicolumn{1}{c}{ Monto } \\
\hline Terreno & 3166667 \\
Edificación materiales & 5039934 \\
Mano de obra & 1800000 \\
$\begin{array}{l}\text { Permisos de construcción y } \\
\text { planos }\end{array}$ & 1025990 \\
$\begin{array}{l}\text { Imprevistos 10\% } \\
\text { (edificaciones) }\end{array}$ & 632693 \\
$\begin{array}{l}\text { Equipo y maquinaria } \\
\text { Permisos para la producción }\end{array}$ & 1550010 \\
Vehículo & 40000000 \\
Trampas para pescar & 400000 \\
Total & $\mathbf{2 3 6 6 2 ~ 8 9 5}$ \\
\hline
\end{tabular}

En total se requieren 23662895 colones para iniciar operaciones. $\mathrm{Su}$ financiamiento debería costearse con recursos provenientes de la cooperación internacional, responsabilidad social empresarial, así como partidas gestionados ante el gobierno local, la Dirección Nacional de Desarrollo de la Comunidad (DINADECO), o bien, fondos para grupos comunitarios del Instituto Mixto de Ayuda Social (IMAS). No se recomienda que su financiamiento se obtenga con base en un empréstito, dado que la actividad productiva aún no ha iniciado y se requiere avanzar en la consolidación del grupo administrador y el modelo de negocios a operar.

Suponiendo la venta de los 30 sacos semanales de harina, a un costo de 20000 colones cada uno, la idea productiva estaría generando ingresos aproximados por 2400000 colones mensuales, teniendo una estructura de costos de operación de 1895000 colones. Por lo que se estima una ganancia aproximada de 505000 colones por mes. Dichas ganancias se estarían repartiendo en partes iguales entre las comunidades participantes en su administración.

La tasa interna de retorno a 7 años se calcula en $2 \%$. Es decir, el rendimiento que haría cero la inversión es de un $2 \%$. Mientras que el valor actual neto de los ingresos, con una tasa de descuento de 5\%, es de $\$ 35729676$. Es decir, el flujo de efectivo descontado y trasladado al poder adquisitivo actual sería de ф35 729676 .

A los cálculos estimados se plantean 4 escenarios (Cuadro 4), el escenario 1 y 4 son positivos, dado que aumentan los ingresos esperados 
y reducen la tasa de descuento, respectivamente. En el primer escenario, se observa que con una modificación de un $20 \%$ en los ingresos en todo el periodo de análisis, la Taza Interna de Retorno (TIR) asume un valor de $2.4 \%$ y el Valor Actual Neto (VAN) de $£ 42875612$. En el cuarto, se reduce la tasa de descuento en un $50 \%$, pasando de $5 \%$ a $2.5 \%$, con estos supuestos, el VAN aumenta a $\notin 38878573$. Por su parte, los escenarios 2 y 3 , se consideran adversos para el proyecto. El escenario 2, se presenta un aumento de un $20 \%$ en la inversión, lo que ajusta a la baja la TIR, al disminuir a $1.47 \%$, y la VAN merma en $\notin 7145$ 935, con respecto a la estimación base. Por último, el escenario 3, presenta un aumento del $50 \%$ de la tasa de descuento, generando una disminución del $7.9 \%$ en el valor actual neto de los ingresos.
El proyecto como tal debe analizarse desde la perspectiva financiera y social. Adicional a la ganancia generada, la tasa interna de retorno obtenida y el valor actualmente calculado, debe recordarse que se estaría brindando empleo a personas de la misma comunidad y aportando en el control de la especie exótica, que tiene presencia abundante en el humedal.

\section{CONCLUSIONES}

El desarrollo de una iniciativa económica que consiste en la utilización del pez pleco como insumo productivo, es un proyecto dirigido a mejorar en la solución de los problemas bilógicos y socioeconómicos identificados en la comunidad de Caño Negro. Por un lado, permite generar acciones para controlar la población

Cuadro 4. Estimación de escenarios TIR y VAN, con base en modificación de ingresos, costos y tasa de descuento

Table 4. Estimated IRR and NPV scenarios, based on modified income, costs and discount rate

\begin{tabular}{llcc}
\hline & \multicolumn{1}{c}{ Costos } & TIR & VAN \\
\hline Escenario base & Estimado base & $2 \%$ & 35729677 \\
Escenario 1 & $+20 \%$ Ingresos & $2.41 \%$ & 42875612 \\
Escenario 2 & $+20 \%$ Inversión inicial & $1.47 \%$ & 28583741 \\
Escenario base & Tasa descuento base & $5 \%$ & 35729677 \\
Escenario 3 & Tasa descuento & $7.5 \%$ & 32924170 \\
Escenario 4 & Tasa descuento & $2.5 \%$ & 38878573 \\
\hline
\end{tabular}


de la especie exótica en el humedal, y por otro, crear opciones de empleo para una comunidad con una alta vulnerabilidad social.

El aprovechamiento mercantil más viable, que utilice como insumo la especie invasora pez pleco en el humedal de Caño Negro, es la producción de harina de pescado para consumo animal. Por tanto, es un proyecto con menor complejidad, en relación con las opciones analizadas; fue seleccionada siguiendo criterios técnicos y aprobada mediante un proceso participativo por el grupo de vecinos que estarían a cargo de la operación de la fábrica. Así mismo, requiere una inversión inicial relativamente baja en infraestructura, maquinaria, equipo y capacitación.

El proyecto es técnicamente viable. Posee una sólida justificación técnica. El estudio de mercado valida la aceptación futura de la harina de pescado para consumo animal y proporciona información inestimable para la determinación del tamaño de escala requerido para la planta, así como otros elementos relacionados con la producción. El estudio técnico determina que es viable según las consideraciones realizadas.

El proyecto debería ser desarrollado en al menos dos etapas. En la primera etapa, se recomienda realizar paralelamente la búsqueda de recursos para la constitución de la fábrica donde se realizará la producción de harina de pescado en la comunidad de Caño
Negro, en conjunto con la selección y capacitación del personal que estará a cargo de ella. La segunda etapa, será la puesta en marcha de la fábrica de harina para consumo animal con base en el pez pleco.

El componente legal frena la ejecución del proyecto, dado que en la actualidad solo se permite la pesca por medios artesanales, y el pez pleco no puede ser capturado mediante estos mecanismos. La modificación de la Ley, debería y podría generarse en el mediano plazo.

El proyecto es financieramente viable. Posee una estructura de costos simple y una expectativa de ventas razonable para el primer año de operación. Proyecta ganancias, genera empleo y apunta a controlar la sobrepoblación de peces plecos en el humedal de Caño Negro. Para su financiamiento original se recomienda realizarlo por medio de cooperación internacional o el apoyo de fondos no reembolsables.

\section{AGRADECIMIENTO}

Se agradece profundamente a los participantes de las Asociaciones de Desarrollo Integral de las comunidades de Veracruz, Los Ángeles, Nueva Esperanza y Caño Negro. Así como la colaboración de Yoselin Ramírez Bravo, por el apoyo en la realización del trabajo de campo y coordinación de actividades del proyecto, a Marco Otoya 
Chavarría, Miriam Miranda Quirós, Jacklyn Rivera Wong y Juan Manuel Herrera Zeledón por los valiosos aportes en el desarrollo de la investigación, así como a las valiosas recomendaciones de los evaluadores de la Revista de Ciencias Marinas y Costeras.

\section{BIBLIOGRAFÍA}

Angulo, A., Garita-Alvarado, C. A., Bussing, W. A. \& López, M. (2013). Annotated checklist of the freshwater fishes of continental and insular Costa Rica: additions and nomenclatural revisions. Check List, 9(5), 987-1019. https://doi. org/10.15560/9.5.987

Angulo, A. (2013). Nombres comunes y técnicos de los peces de agua dulce de Costa Rica. Rev. Fil. Lin. UCR, 39(2), 77-103.

Arroyo, M. (2008). Aprovechamiento de la harina de plecostomus spp. como ingrediente en alimento para el crecimiento de tilapia (Oreochromis niloticus). Tesis de maestría no publicada, México.

Ayala-Pérez, L., Pineda-Peralta, A., Álvarez-Guillen, H. \& Amador-del Ángel, L. (2014). El pez diablo (Pterygoplichthys spp.) en las cabeceras estuarinas de la Laguna de Términos, Campeche. En A., Low, P. Quijón \& E. Peters (Eds.). Especies invasoras acuáticas: casos de estudio en ecosistemas de México (pp. 313-336), México: University of Prince Edward Island.

Blackburn, T., Pysek, P., Bacher, S., Carlton, J., Duncan, R., Jarosik, V., Wilson, J. \& Richardson, D. (2011) A proposed unified framework for biological invasions. Trends Ecol. Evol., 26(7), 333-339. https://doi.org/10.1016/j. tree.2011.03.023
Castellón, D. (2017). Consume pez Diablo. Recuperado en marzo 15, 2017, disponible en https://www.sdpnoticias. com/nacional/2017/01/13/consume-pez-diablo

Chacón, D. (2016). Informe final sobre el estado del conocimiento de la invasión del pez pleco (Hypostomus aspidolepis) y recomendaciones para su control en el humedal protegido de importancia internacional Caño Negro. San José, Costa Rica: Sin editorial.

Corea, J., Hernández, G., Solís, V. \& Aguilar A. J. (2014). Distribución y abundancia de peces de la familia Loricariidae (Pleco) y su relación con los peces de interés comercial en los alrededores de la Isla de Ometepe. Encuentro, 98, 4459. https://doi.org/10.5377/encuentro. v0i98.1445

Covain, R. \& Fisch-Muller, S. (2007). The genera of the Neotropical armored catfish subfamily Loricariinae (Siluriformes: Loricariidae): a practical key and synopsis. Zootaxa, 1462, 1-40.

El Nuevo Diario. (2010). Nicaragua a la pesca del Pez Diablo que afecta Guatemala. Recuperado en marzo 12, 2017, disponible en https://www.elnuevodiario. com.ni/nacionales/81538-nicaragua-pesca-pez-diablo-que-afecta-guatemala/

Escalera, C., Aroyo, M., Moncayo, R. \& Zarazúa, J. (2012). Pesquería sustentable y desarrollo local. Uso y aprovechamiento potencial del Pez Diablo. Desarrollo Local y Empresas 32(2), 39-58.

Guerrero, T. (2016). Propuesta base para la estrategia de manejo y control local del pez diablo en el Refugio Nacional de Vida Silvestre mixto Caño Negro, Costa Rica. Tesis de maestría no publicada. CATIE

Herrera, D. \& Molina, A (2011). Peces Diablo (Teleósteo: Siluriformes: Loricariidae) 
en la cuenca del Río Reventazón, Costa Rica. Biocenosis, 25(1-2), 79-86.

INEC. Instituto Nacional de Estadísticas y Censos. (2015). Encuesta Nacional a Hogares. Recuperado el 20 febrero 2017. Costa Rica: INEC, Recuperado de http://www.inec.go.cr/encuestas/encuesta-nacional-de-hogares

INEC. Instituto Nacional de Estadísticas y Censos. (2017). Censos 2011. Indicadores demográficos y sociales. Costa Rica: INEC. Recuperado en febrero 20, 2017, disponible en http://www.inec. go.cr/censos/censos

Informador. (2017). Pez diablo, ángel de la nutrición. Recuperado en marzo 25, 2017 , disponible en http://www.informador. com.mx/tecnologia/2011/319848/6/ pez-diablo-angel-de-la-nutricion.htm

Maldonado, E., López, U., Salinas, R., González, N., Cuenca, C., Jiménez, R. \& Hernández, J. (2015). Contenido de metales pesados en músculo de pez diablo Pterygoplichthys pardalis. Rev. Iberoam. Ciencias, 2(1), 1-7.

Martinez, M. (2007). Especies invasoras de alto impacto a la biodiversidad: Prioridades en México. México: Instituto Mexicano de Tecnología del Agua.

Medina, M. (2017). Análisis de los emprendimientos implementados en el área marina protegida manglares el morro y su incidencia en el desarrollo sostenible. Guayaquil, Ecuador: Universidad de Guayaquil.

Mendoza, R., Contreras, S., Ramírez, C., Koleff, P., Álvarez, P. \& Aguilar, V. (2007). Los peces diablo. Biodiversitas, 70, 1-5.

MIDEPLAN. Ministerio de Planificación Nacional y Política Económioca. (2017). Índice de Desarrollo Social (IDS), distrital. Recuperado en enero 18, 2017, disponible en https://documentos.mideplan.go.cr/share/s/612bxJTjQqWPGoLkFtG9Xw
Milenio, G. (2017). Pez diablo ¿Medicinal? Recuperado en febrero 20, 2017, disponible en http://www.milenio.com/ tendencias/pez_diablopez_diablo_medicinal_0_697730456.html

Nico, L., Loftus, W. \& P. Reid. (2009a). Interactions between non-native armored suckermouth catfish (Loricariidae: Pterygoplichthys) and native Florida manatee (Trichechus manatus latirostris) in artesian springs. Aquat. Invas., 4(3), 511-519. https://doi.org/10.3391/ ai.2009.4.3.13

Nico, L., Howard, J. \& Tuten, T. (2009b). Non-native suckermouth armored catfishes in Florida: Description of nest burrows and burrow colonies with assessment of shorline condictions. ANSRP Bull., 9(1), 1-30.

Pedraza, O. (2011). Modelo del plan de negocios. México: Grupo Editorial Patria.

PNUD. Programa de las Naciones Unidas para el Desarrollo. (2015). Atlas del desarrollo humano cantonal de Costa Rica. San José, Costa Rica: PNUD.

PRODAPESI. Programa para la Disminución y el Aprovechamiento de Especies Invasoras. (s. f.). Elaboración de harina de Plecostomus: Manual de Apoyo. México: PRODAPESI.

Proyecto Humedales. (2017). Proyecto conservación, uso sostenible de la biodiversidad y mantenimiento de los servicios de los ecosistemas de humedales protegidos de importancia internacional. Recuperado en marzo 25, 2017, disponible en http://www.proyectohumedalescr.info/2016/01/quienes-somos.html Rodríguez-Santiago, M. A., García-Prieto, L., Mendoza-Garfias, B., González-Solís, D. \& Grano-Maldonado, M. (2016). Parasites of two coexisting invasive sailfin catfishes (Siluriformes: Loricariidae) in a tropical region of Mexico. Neotrop. Ichthyol., 14(3), 1-7. https:// doi.org/10.1590/1982-0224-20160021 
Sanz, B. (2010). Cryptosporidium y Toxoplasma. Dos importantes protozoos parásitos transmisibles por los alimentos y el agua. En Real Académia de Farmacia (Ed.), Monografía XXXI: Aspectos higiénicos de los alimentos microbiológicamente seguros (pp. 219-301). Madrid, España: Real Academia Nacional de Farmacia.

Sapag, N. (2007). Proyectos de inversión. Formulación y evaluación. México: Pearson.

Tobón, S. (2008). La formación basada en competencias en la educación superior: el enfoque complejo. Guadalajara, México: Universidad Autónoma de Guadalajara.

Valdez, J. (2011). Revisión y propuesta de proyecto de una planta productora de harina de pescado. Tesis no publicada de grado, Instituto Tecnológico de Tuxtla Gutíerrez, México.
Valverde, J. \& Alfaro, J. (2014). Productividad y rentabilidad del cultivo de camarones marinos en el Golfo de Nicoya, Costa Rica . Rev. Cien. Mar. Cost., 6, 37-53. https://doi.org/10.15359/revmar.6.3

Wakida-Kusunoki, A. \& Amador del Angel, L. E. (2008). Nuevos registros de los plecos Pterygoplichthys pardalis (Castelnau 1855) y P. disjunctivus (Weber 1991) (Siluriformes: Loricariidae) en el Sureste de México. Hidrobiológica, 18(3), 251-256.

Zequeira, M., Varona, S. \& Figueredo, E. (2017). Factibilidad económica del cambio de uso del suelo en áreas cubiertas por marabú en la CCSF "Francisco Borrero Lavadí” de Camagüey. En C. Gómez \& P. Pérez (Eds.). Evaluaciones económicas de especies exóticas invasoras (pp. 100-128). La Habana, Cuba: Centro Nacional de Áreas Protegidas Centro Nacional de Áreas Protegidas. 